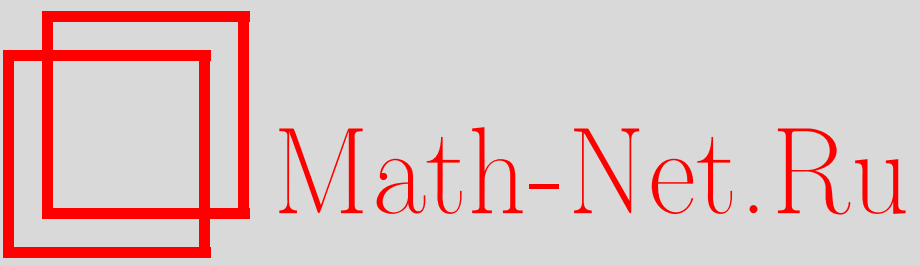

В. С. Матвеев, П. Ж. Топалов, Геодезическая эквивалентность метрик как частный случай интегрируемости геодезических потоков, ТМФ, 2000, том 123, номер 2, 285-293

DOI: https://doi.org/10.4213/tmf602

Использование Общероссийского математического портала Math-Net.Ru подразумевает, что вы прочитали и согласны с пользовательским соглашением

http://www.mathnet.ru/rus/agreement

Параметры загрузки:

IP : 54.80 .73 .141

26 апреля 2023 г., 13:14:12 
ТЕОРЕТИЧЕСКАЯ

И МАТЕМАТИЧЕСКАЯ

ФИЗИКА

Том 123, № 2

май, 2000

(C) 2000 г.

В. С. Матвеев*, П. Ж. Топалов ${ }^{\dagger}$

\section{ГЕОДЕЗИЧЕСКАЯ ЭКВИВАЛЕНТНОСТЬ МЕТРИК КАК ЧАСТНЫЙ СЛУЧАЙ ИНТЕГРИРУЕМОСТИ ГЕОДЕЗИЧЕСКИХ ПОТОКОВ}

Рассмотрена связь между геодезически эквивалентными метриками и интегрируемыми геодезическими потоками. Если две различные метрики на одном многообразии имеют одинаковые геодезические, то геодезические потоки этих метрик допускают достаточно много интегралов (специального вида) в инволюции и наоборот. Существует также квантовый вариант этого результата: если две метрики на одном многообразии имеют одинаковые геодезические, то оператор Бельтрами-Лапласа $\Delta$ для каждой метрики допускает достаточно много линейных дифференциальных операторов, коммутирующих с $\Delta$. Отсюда следует, что топология многообразия (с двумя различными метриками с одинаковыми геодезическими) должна быть достаточно проста. Кроме того, из непропорциональности метрик в точке следует непропорциональность метрик почти во всех точках.

Памяти Михаила Владимировича Савельева

Приведем основное для нас определение. Пусть $g=\left(g_{i j}\right)$ и $\bar{g}=\left(\bar{g}_{i j}\right)$ - гладкие метрики на одном и том же многообразии $M^{n}$.

ОПРЕДЕЛЕНИЕ 1 . Метрики $g$ и $\bar{g}$ геодезически әквивалентны, если они имеют одни и те же геодезические (рассматриваемые как непараметризованные кривые).

В 1869 г. Дини [1] сфформулировал задачу локальной классификации геодезически эквивалентных метрик и решил ее в размерности два. В 1896 г. Леви-Чивита [2] получил локальное описание геодезически эквивалентных метрик на многообразиях произвольных размерностей. Многие интересные результаты в этой области получили Пенлеве, Вейль, Картан, Широков, Кобаяши, Синюков, Петров, Вензи, Микеш, Аминова (см. библиографию в $[3,4])$.

Предлагаемый нами подход помогает ответить на следующие вопросы:

1. Какие замкнутые многообразия допускают геодезически эквивалентные метрики $[5]$ ?

\footnotetext{
* Челябинский государственный университет, Челябинск, Россия.

E-mail: matveev@mail.cgu.chel.su

${ }^{\dagger}$ Department of Differential Equations, Institute of Mathematics and Informatics, BAS, Sofia, Bulgaria. E-mail: topalov@math.bas.bg
} 
2. Сколько имеется метрик, геодезически эквивалентных данной [4]?

Вначале приведем два простых примера геодезически эквивалентных метрик на замкнутых многообразиях. Всегда сушествует простейший пример, который не интересен для нашего рассмотрения: произвольная метрика $g$ геодезически эквивалентна метрике $C g$, где $C$ - постоянная.

Первый нетривиальный в этом смысле пример следующий. Возьмем тор $T^{n}=S^{1} \times$ $S^{1} \times \cdots \times S^{1}$ и рассмотрим систему координат $x^{1}, \ldots, x^{n}$ на $T^{n}$, где $x^{k}-$ циклическая координата на $k$-й окружности. Теперь рассмотрим метрики

$$
d s_{1}^{2}=\sum_{i=1}^{n}\left(d x^{i}\right)^{2}, \quad d s_{2}^{2}=\sum_{i, j=1}^{n} a_{i j}\left(d x^{i}\right)\left(d x^{j}\right),
$$

где $\left(a_{i j}\right)$ - положительно-определенная симметричная матрица. Очевидно, что метрики $d s_{1}^{2}$ и $d s_{2}^{2}$ геодезически эквивалентны и, вообще говоря, не пропорциональны.

Второй нетривиальньй пример принадлежит Бельтрами $[6,7]$. Рассмотрим сферу

$$
\sum_{i=1}^{n+1}\left(x^{i}\right)^{2}=1
$$

где $x^{1}, \ldots, x^{n+1}$ - стандартные координаты в евклидовом пространстве $R^{n+1}$. Возьмем линейное преобразование $L: R^{n+1} \rightarrow R^{n+1}$ пространства $R^{n+1}$ и рассмотрим соответствуюшее проективное преобразование $l$ сферы. Пусть $x$ - точка на сфере. Рассмотрим луч $[0, x)$, где 0 - начало координат в $R^{n+1}$. Очевидно, что образ $L([0, x))$ также является лучом с началом в нуле. Пусть луч $L([0, x))$ и сфера пересекаются в точке $y$. По определению положим $l(x)=y$. Другими словами, отображение $l$ дается формулой

$$
l(x)=\frac{L x}{\|L x\|} .
$$

Легко видеть, что отображение $l$ сохраняет геодезические на сфере. Действительно, геодезические на сфере являются пересечениями со сферой плоскостей, которые проходят через начало координат. Линейное отображение $L$ переводит плоскости в плоскости, следовательно, проективное отображение $l$ переводит геодезические в геодезические. Тогда стандартная метрика $g_{\mathrm{st}}$ на сфере и метрика $l^{*} g_{\mathrm{st}}$ являются геодезически эквивалентными.

Отметим следуюшее обшее свойство этих двух примеров: геодезические потоки метрик полностью интегрируемы в том смысле, что существует $n$ интегралов в инволюции. Основной результат данной работы заключается в том, что указанное свойство является обшим для всех геодезически эквивалентных метрик общего положения.

Обозначим через $G$ линейный оператор $g^{-1} \bar{g}=\left(g^{i \alpha} \bar{g}_{\alpha j}\right)$. Другими словами, оператор $G$ "переводит" метрику $\bar{g}$ в $g$ : для произвольных векторов $\nu, \xi \in T_{x_{0}} M^{n}$ скалярное произведение $\bar{g}(\xi, \nu)$ векторов $\xi$ и $\nu$ в метрике $\bar{g}$ равно скалярному произведению $g(G \xi, \nu)$ векторов $G \xi$ и $\nu$ в метрике $g$. 
Рассмотрим характеристический многочлен

$$
\operatorname{det}(G-\mu E)=c_{0} \mu^{n}+c_{1} \mu^{n-1}+\cdots+c_{n} .
$$

Коэффициенты $c_{1}, \ldots, c_{n}$ являются гладкими функциями на многообразии $M^{n}$, а $c_{0} \equiv$ $(-1)^{n}$. Рассмотрим функции $I_{k}: T M^{n} \rightarrow R, k=0, \ldots, n-1$, задаваемые обшей формулой

$$
I_{k}(\xi)=\left(\frac{\operatorname{det}(g)}{\operatorname{det}(\bar{g})}\right)^{\frac{k+2}{n+1}} g\left(S_{k} \xi, \xi\right),
$$

где $S_{k}$ - линейный оператор:

$$
S_{k} \stackrel{\text { def }}{=} \sum_{i=0}^{k} c_{i} G^{k-i+1},
$$

a $g(\nu, \xi)$ обозначает скалярное произведение касательных векторов $\nu$ и $\xi$ в метрике $g$.

Теорема 1 [8]. Если метрики g и $\bar{g}$ на $M^{n}$ геодезически эквивалентны, то функции $I_{k}$ являются интегралами геодезических потоков метрики $g$ и попарно коммутируют (если мы отождествим с помощью $g$ касательное и кокасательное расслоения над $M^{n}$ и рассмотрим каноническую симплектическую форму на кокасательном расслоении).

ЗАМЕЧАНИЕ 1. Интеграл

$$
I_{0}=\left(\frac{\operatorname{det}(g)}{\operatorname{det}(\bar{g})}\right)^{\frac{2}{n+1}} \bar{g}(\xi, \xi)
$$

был получен Пенлеве [2]. Интеграл $I_{n-1}$ есть интеграл энергии (умноженный на минус два). Интегралы $I_{1}, I_{2}, \ldots, I_{n-2}$, по-видимому, являются новыми.

ЗАмЕчАниЕ 2. В двумерном случае теорема 1 следует из результатов Пенлеве [2].

ЗАмечание 3 [8]. Метрики $g, \bar{g}$ строго непропорииональны, если характеристический многочлен $\operatorname{det}(G-\mu E)$ не имеет кратных корней. Легко показать, что если метрики $g, \bar{g}$ геодезически эквивалентны и строго непропорциональны в некоторой точке, то в касательном расслоении достаточно малой окрестности этой точки интегралы $I_{k}$ почти всюду функционально независимы.

Теорема 1 позволяет приложить теорию интегрируемых геодезических потоков к теории геодезически эквивалентных метрик и наоборот.

СледСтвиЕ 1. Предположим, что многообразие $M^{n}$ связно. Пусть метрики $g, \bar{g}$ на $M^{n}$ геодезически эквивалентны и строго непропорииональны, по крайней мере, в одной точке на $M^{n}$. Тогда метрики строго непропорциональны почти всюдуна $M^{n}$. 
ТеОрема 2. Пусть $g, \bar{g}$ - метрики на многообразии $M^{n}$. Пусть функции $I_{0}$, $I_{1}, \ldots, I_{n-1}$ почти всюду функционально независимы и коммутируют (если мы отождествим с помощью $g$ касательное и кокасательное расслоения над $M^{n} u$ рассмотрим каноническую симплектическую форму на кокасательном расслоении). Тогда метрики $g$ и $\bar{g}$ геодезически әквивалентны.

ЗАмЕчАниЕ 4. В двумерном случае теорема 2 была сформулирована в книге [9].

ТЕОрема 3 [10]. Если вещественно-аналитическое замкнутое многообразие $M^{n}$ с вещественно-аналитической метрикой удовлетворяет, по крайней мере, одному из условий: а) группа $\pi_{1}\left(M^{n}\right)$ не является почти коммутативной; б) $\operatorname{dim} H_{1}\left(M^{n} ; \mathbf{Q}\right)>\operatorname{dim} M^{n}$, то геодезический поток на $M^{n}$ не является аналитически интегрируемым.

СЛЕДСТВИЕ 2 [8]. Пусть $M^{n}$ - замкнутое вещественно-аналитическое многообразие, снабженное двумя вещественно-аналитическими метриками $g$ и $\bar{g}$, которые геодезически әквивалентны и строго непропорчиональны, по крайней мере, в одной точке. Тогда фундаментальная группа $\pi_{1}\left(M^{n}\right)$ содержит коммутативную подгруппу конечного индекса, а размерность гомологической группь $H_{1}\left(M^{n} ; \mathbf{Q}\right)$ не превосходит $n$.

СЛЕДСТВИЕ 3 [8]. Пусть метрики $g$ и $\bar{g}$ на замкнутой поверхности с отрицательной әйлеровой характеристикой геодезически әквивалентны. Тогда $g=C \bar{g}$, где $C$ - константа.

СлЕДСТВИЕ 4 [8]. Пусть метрики $g$ и $\bar{g}$ на торе $T^{2}$ геодезически әквивалентны. Если они пропорииональны в точке $x \in T^{2}$, то $g=C \bar{g}$, где $C$ - положительная постоянная.

СЛЕДСТВИЕ 5 [8]. Пусть метрики $g$ и $\bar{g}$ на сфере $S^{2}$ геодезически әквивалентны. Тогда имеются только три возможности:

а) метрики пропорииональнь ровно в двух точках;

б) метрики пропорииональны ровно в четырех точках;

в) метрики полностью пропоричональнь, т.е. $g=C \bar{g}$, где $C$ - положстельная постоянная.

В первом случае метрики допускают векторное поле Киллинга.

Приведем конструкцию, которая по орбитальному диффеоморфизму между двумя гамильтоновыми системами дает их интегралы.

Пусть $v$ и $\bar{v}$ - гамильтоновы системы на симплектических многообразиях $(N, \omega)$ и $(\bar{N}, \bar{\omega})$ с гамильтонианами $H$ и $\bar{H}$ соответственно. Рассмотрим изоэнергетические поверхности

$$
Q \stackrel{\text { def }}{=}\{x \in N: H(x)=h\}, \quad \bar{Q} \stackrel{\text { def }}{=}\{x \in \bar{N}: \bar{H}(x)=\bar{h}\},
$$

где $h$ и $\bar{h}$ - регулярные значения соответственно функций $H$ и $\bar{H}$. 
ОПРЕДЕЛЕниЕ 2. Говорят, что дифффеоморфизм $\phi: Q \longrightarrow \bar{Q}$ орбитальный, если он переводит орбиты системы $v$ в орбиты системы $\bar{v}$.

Для данного орбитального диффеоморфизма мы можем инвариантным образом построить интегралы. Обозначим через $\sigma$ и $\bar{\sigma}$ ограничение форм $\omega$ и $\bar{\omega}$ соответственно на $Q$ и $\bar{Q}$. Рассмотрим форму $\phi^{*} \bar{\sigma}$ на $Q$.

Лемма 1 [11]. Поток $v$ сохраняет форму $\phi^{*} \bar{\sigma}$.

ДоКАЗАТЕЛЬСТВо. Производная Ли $L_{v}$ формы $\phi^{*} \bar{\sigma}$ вдоль векторного поля $v$ удовлетворяет уравнению

$$
L_{v} \phi^{*} \bar{\sigma}=\mathrm{d}\left[\imath_{v} \phi^{*} \bar{\sigma}\right]+\imath_{v} \mathrm{~d}\left[\phi^{*} \bar{\sigma}\right] .
$$

Оба члена в правой части равны нулю. Поскольку форма $\bar{\omega}$ замкнута, форма $\bar{\sigma}$ также замкнута и $\mathrm{d}\left[\phi^{*} \bar{\sigma}\right]=\phi^{*}(\mathrm{~d} \bar{\sigma})=0$. Так как диффеоморфизм переводит орбиты в орбиты, он переводит ядро формы $\sigma$ в ядро формы $\bar{\sigma}$, так что $\imath_{v} \phi^{*} \bar{\sigma}=0$. Лемма 1 доказана.

Очевидно, что ядра форм $\sigma$ и $\phi^{*} \bar{\sigma}$ совпадают (в пространстве $\mathcal{T}_{x} Q$ в каждой точке $x \in Q)$ с линейной оболочкой вектора $v$. Следовательно, эти формы индуцируют два невырожденных тензорных поля на фактор-расслоении $\mathcal{T} Q /\langle v\rangle$. Обозначим соответствуюшие формы на $\mathcal{T} Q /\langle v\rangle$ также буквами $\sigma, \bar{\sigma}$.

Лемма 2. Характеристический многочлен оператора $(\sigma)^{-1}\left(\phi^{*} \bar{\sigma}\right)$ на $\mathcal{T} Q /\langle v\rangle$ сохраняется потоком $v$.

ДоКАЗАТЕЛЬСТВо. Поскольку поток $v$ сохраняет гамильтониан $H$ и форму $\omega$, он сохраняет и форму $\sigma$. Так как поток $v$ сохраняет обе формы, он сохраняет характеристический многочлен оператора $(\sigma)^{-1}\left(\phi^{*} \bar{\sigma}\right)$. Лемма 2 доказана.

Поскольку обе формы являются кососимметричными, каждый корень характеристического многочлена оператора $(\sigma)^{-1}\left(\phi^{*} \bar{\sigma}\right)$ имеет четную кратность. Тогда характеристический многочлен представляет собой квадрат многочлена $\delta^{n-1}(t)$ степени $n-1$. Поэтому многочлен $\delta^{n-1}(t)$ также сохраняется потоком $v$. Таким образом, коэффициенты многочлена $\delta^{n-1}(t)$ являются интегралами системы $v$.

Геодезические потоки геодезически эквивалентных метрик можно рассматривать как орбитально эквивалентные системы. Многообразие $N=\bar{N}=T M^{n}$, формы $\omega, \bar{\omega}$ определяются как

$$
\omega \stackrel{\text { def }}{=} \mathrm{d}\left[g_{i j} \xi^{j} d x^{i}\right], \quad \bar{\omega} \stackrel{\text { def }}{=} \mathrm{d}\left[\bar{g}_{i j} \xi^{j} d x^{i}\right],
$$

а орбитальный диффеоморфизм ф задается соотношением

$$
\phi(x, \xi)=\left(x, \xi \frac{\|\xi\|_{g}}{\|\xi\|_{\bar{g}}}\right) .
$$

Прямыми вычислениями мы получаем формулу для интегралов $I_{k}$ из теоремы 1.

Рассмотрим следующий вопрос: есть ли интересные примеры геодезически эквивалентных метрик на замкнутых многообразиях? Из приведенной ниже теоремы 4 (принадлежащей главным образом Синюкову [12]) можно получить конструкцию, которая 
по паре геодезически эквивалентных метрик дает другую пару геодезически эквивалентных метрик. Начиная с метрики постоянной кривизны на сфере мы получаем метрику эллипсоида и метрику пуассоновой сферы.

Пусть $g, \bar{g}$ - римановы метрики на многообразии $M^{n}$. Рассмотрим линейньй оператор $B: T M^{n} \rightarrow T M^{n}$, задаваемый как

$$
B_{j}^{i}=\left(\frac{\operatorname{det}(\bar{g})}{\operatorname{det}(g)}\right)^{\frac{1}{n+1}} \bar{g}^{i p} g_{p j}
$$

По определению положим метрику $g B$ равной $g_{i p} B_{j}^{p}$, метрику $\bar{g} B$ равной $\bar{g}_{i p} B_{j}^{p}$. Другими словами, произведение $g B(\xi, \nu)$ произвольных векторов $\xi, \nu \in T_{x_{0}} M^{n}$ равно $g(B \xi, \nu)$, а скалярное произведение $\bar{g} B(\xi, \nu)$ равно $\bar{g}(B \xi, \nu)$. Очевидно, что линейный оператор $B$ самосопряжен относительно метрик $g$ и $\bar{g}$, а метрики $g B$ и $\bar{g} B$ корректно определены.

ТЕОремА 4. Метрики $g$ и $\bar{g}$ геодезически әквивалентны тогда и только тогда, когда метрики $g B$ и $\bar{g} B$ геодезически әквивалентны.

Ясно, что метрики $g$ и $\bar{g}$ строго непропорциональны (почти всюду) тогда и только тогда, когда метрики $g B$ и $\bar{g} B$ строго непропорциональны (почти всюду). Таким образом, если мы имеем пару геодезически эквивалентных метрик $g$ и $\bar{g}$, то можем построить другую пару геодезически эквивалентных метрик. Применяя это построение еще раз, мы получаем еще одну пару геодезически эквивалентных метрик. Естественно обозначить ее через $g B^{2}, \bar{g} B^{2}$, поскольку

$$
g B^{2}(\xi, \nu)=g\left(B^{2} \xi, \nu\right), \quad \bar{g} B^{2}(\xi, \nu)=\bar{g}\left(B^{2} \xi, \nu\right) .
$$

Можно действовать по-другому и рассмотреть метрики $g B^{-1}$ и $\bar{g} B^{-1}$, задаваемые как

$$
g B^{-1}(\xi, \nu)=g\left(B^{-1} \xi, \nu\right), \quad \bar{g} B^{-1}(\xi, \nu)=\bar{g}\left(B^{-1} \xi, \nu\right) .
$$

Они также геодезически эквивалентны.

Для начала этого процесса требуется исходная пара геодезически эквивалентных метрик $g$ и $\bar{g}$. Возьмем пару $g_{\mathrm{st}}$ и $l^{*} g_{\mathrm{st}}$ из второго примера. Тогда метрика $g B$ является метрикой эллипсоида, а $\bar{g} B^{2}$ - метрикой пуассоновой сферы. Варьируя линейньй оператор $L$, можно получить метрики всех возможных эллипсоидов и пуассоновых сфер.

ТЕОРЕма 5 (независимо полученная Табачниковым[13]). Ограничение евклидовой метрики

$$
\left(d x^{1}\right)^{2}+\cdots+\left(d x^{n+1}\right)^{2}
$$

на стандартный әллипсоид

$$
E^{n}=\left\{\left(x^{1}, x^{2}, \ldots, x^{n+1}\right) \in R^{n+1}: \frac{\left(x^{1}\right)^{2}}{a_{1}}+\cdots+\frac{\left(x^{n+1}\right)^{2}}{a_{n+1}}=1\right\}
$$

геодезически әквивалентно ограничению метрики

$$
\frac{1}{\left(\frac{x^{1}}{a_{1}}\right)^{2}+\cdots+\left(\frac{x^{n+1}}{a_{n+1}}\right)^{2}}\left(\frac{\left(d x^{1}\right)^{2}}{a_{1}}+\cdots+\frac{\left(d x^{n+1}\right)^{2}}{a_{n+1}}\right)
$$


на тот же әллипсоид.

Согласно работе [14] метрику пуассоновой сферы можно рассматривать как ограничение метрики

$$
\frac{1}{\frac{\left(x^{1}\right)^{2}}{a_{1}^{2}}+\cdots+\frac{\left(x^{n+1}\right)^{2}}{a_{n+1}^{2}}}\left(\left(d x^{1}\right)^{2}+\cdots+\left(d x^{n+1}\right)^{2}\right)
$$

на эллипсоид

$$
E^{n}=\left\{\left(x^{1}, x^{2}, \ldots, x^{n+1}\right) \in R^{n+1}: \frac{\left(x^{1}\right)^{2}}{a_{1}}+\cdots+\frac{\left(x^{n+1}\right)^{2}}{a_{n+1}}=1\right\} .
$$

Теорема 6 [15]. Оораничение метрики

$$
\frac{1}{\frac{\left(x^{1}\right)^{2}}{a_{1}^{2}}+\cdots+\frac{\left(x^{n+1}\right)^{2}}{a_{n+1}^{2}}}\left(\left(d x^{1}\right)^{2}+\cdots+\left(d x^{n+1}\right)^{2}\right)
$$

на эллипсоид

$$
E^{n}=\left\{\left(x^{1}, x^{2}, \ldots, x^{n+1}\right) \in R^{n+1}: \frac{\left(x^{1}\right)^{2}}{a_{1}}+\cdots+\frac{\left(x^{n+1}\right)^{2}}{a_{n+1}}=1\right\}
$$

геодезически әквивалентно ограничению метрики

$$
a_{1}\left(d x^{1}\right)^{2}+\cdots+a_{n+1}\left(d x^{n+1}\right)^{2}-\left(x^{1} d x^{1}+x^{2} d x^{2}+\cdots+x^{n+1} d x^{n+1}\right)^{2}
$$

на тот же әллипсоид.

Рассмотрим проблему квантовой интегрируемости оператора Бельтрами-Лапласа для геодезически эквивалентных метрик и разделения переменных. Возьмем оператор Бельтрами-Лапласа $\Delta:=g^{i j} \nabla_{i} \nabla_{j}$, где $\nabla$ обозначает ковариантную производную, соответствуюшую метрике $g$. Рассмотрим дифференциальные операторы

$$
\mathcal{I}_{0}, \mathcal{I}_{1}, \ldots, \mathcal{I}_{n-1}: C^{2} \rightarrow C^{0}
$$

задаваемые общей формулой

$$
\mathcal{I}_{k}:=g^{i j} \nabla_{i}\left(\frac{\operatorname{det}(g)}{\operatorname{det}(\bar{g})}\right)^{\frac{k+2}{n+1}}\left(S_{k}\right)_{j}^{p} \nabla_{p}
$$

ЗАмЕЧАНИЕ 5 . Оператор $\mathcal{I}_{n-1}$ есть в точности оператор $-\Delta$.

ТЕОрема 7. Если метрики $g$ и $\bar{g}$ на $M^{n}$ геодезически эквивалентны, то операторь $\mathcal{I}_{k}$ попарно коммутируют. Еслимногообразие $M^{n}$ замкнуто, то операторы $\mathcal{I}_{k}$ являются самосопряжсенными. 
СлеДСтвиЕ 6. Предположим, что многообразие $M^{n}$ связно. Предположим, что метрики $g$ и $\bar{g}$ на $M^{n}$ геодезически эквивалентныр. Они строго непропорциональнь, по крайней мере, в одной точке $M^{n}$ тогда и только тогда, когда операторы $\mathcal{I}_{0}, \ldots, \mathcal{I}_{n-1}$ линейно независимь.

Таким образом, если многообразие замкнуто и если метрики $g$ и $\bar{g}$ на нем геодезически эквивалентны и строго непропорциональны, по крайней мере, в одной точке, то мы имеем полную квантовую интегрируемость оператора Бельтрами-Лапласа метрики $g$.

Очевидно, что символами операторов $\mathcal{I}_{k}$ являются $I_{k}$. Тогда если операторы $\mathcal{I}_{k}$ коммутируют, то и функции $I_{k}$ тоже коммутируют.

СлЕДСтвиЕ 7. Предположим, что многообразие $M^{n}$ связно. Пусть $g$ и $\bar{g}-$ метрики на нем. Пусть операторы $\mathcal{I}_{k}$ коммутируют и пусть они линейно независимы. Тогда метрики $g$ и $\bar{g}$ геодезически эквивалентны.

Квантовая интегрируемость означает, что сушествует счетньй базис

$$
\Phi=\left\{\phi_{1}, \phi_{2}, \ldots, \phi_{m}, \ldots\right\}
$$

в пространстве $L_{2}\left(M^{n}\right)$ такой, что каждое $\phi_{m}$ - это собственная функция каждого оператора $\mathcal{I}_{k}$.

Более того, в нашем случае переменные можно разделить. Точнее, любая функция $\phi$ из базиса $\Phi$ является собственной функцией каждого оператора $\mathcal{I}_{k}$, и поэтому представляет собой решение системы $n$ дифференциальных уравнений в частных производных

$$
\mathcal{I}_{k} \phi=\lambda_{k} \phi, \quad k=0,1, \ldots, n-1 .
$$

Разделение переменных означает, что в окрестности почти любой точки существуют координаты $\left(x^{0}, x^{1}, \ldots, x^{n-1}\right)$ такие, что в этих координатах система (1) эквивалентна системе

$$
\frac{\partial^{2}}{\left(\partial x^{k}\right)^{2}} \phi=F_{k}\left(x^{k}, \lambda_{0}, \lambda_{1}, \ldots, \lambda_{n-1}\right) \phi, \quad k=0,1, \ldots, n-1,
$$

где функции $F_{k}$ зависят от переменной $x^{k}$ и параметров $\lambda_{0}, \lambda_{1}, \ldots, \lambda_{n-1}$. Тогда функция $\phi$ есть произведение

$$
X_{0}\left(x^{0}\right) X_{1}\left(x^{1}\right) \ldots X_{n-1}\left(x^{n-1}\right),
$$

а каждое $X_{k}$ - это решение $k$-го уравнения системы (2), так что мы свели систему дифференциальных уравнений в частных производных (1) к системе обыкновенных дифференциальных уравнений

$$
\frac{\partial^{2}}{\left(\partial x^{k}\right)^{2}} X_{k}\left(x^{k}\right)=F_{k}\left(x^{k}, \lambda_{0}, \lambda_{1}, \ldots, \lambda_{n-1}\right) X_{k}\left(x^{k}\right), \quad k=0,1, \ldots, n-1 .
$$

Каково пространство метрик, геодезически эквивалентных данной? Если две метрики геодезически эквивалентны, то существует, по крайней мере, однопараметрическое семейство геодезически эквивалентных метрик, которое включает эти две метрики [16]. 
Можно показать, что (по крайней мере, в вешественно-аналитическом случае) множество метрик, геодезически эквивалентных данной, является многообразием.

Чему равна размерность этого многообразия? Даже локально существуют метрики, не допускающие (нетривиальных) геодезически эквивалентных метрик [4]. Кроме того, локально размерность этого пространства не превьшает $(n+1)(n+2) / 2$ и равна $(n+1)(n+2) / 2$ только для метрик постоянной кривизны [4].

Пусть $M^{n}$ замкнуто и пусть $g$ и $\bar{g}$ геодезически эквивалентны и строго непропорциональны на почти всюду плотном множестве точек. Тогда геодезический поток метрики $g$ полностью интегрируем, а почти все орбиты лежат на соответствующих торах Лиувилля. Предположим, что геодезический поток является нерезонансным. Тогда слоение Лиувилля однозначно определено и все интегралы геодезического потока коммутируют с интегралами $I_{0}, \ldots, I_{n-1}$. Кроме того, примем, что у торов Лиувилля геодезического потока имеется достаточное количество каустик: почти каждая точка поверхности является пересечением $n$ каустик. Тогда размерность пространства метрик (по модулю умножения на константу), геодезически эквивалентных метрике $g$, равна единице.

Геодезические потоки метрик эллипсоида и пуассоновой сферы удовлетворяют всем этим условиям.

Благодарности. Работа частично поддержана $\mathrm{MESC}^{\prime}$, грант № MM-810/98.

\section{Список литературы}

[1] U. Dini. Ann. Math. Ser. 2. 1869. V. 3. P. 269-293.

[2] T. Levi-Civita. Ann. Mat. Ser. $2^{a}$. 1896. V. 24. P. 255-300.

[3] А.В. Аминова. УМН. 1993. Т. 48. № 2. С. 107-164.

[4] J. Mikesh. J. Math. Sci. 1996. V. 78. № 3. Р. 311-333; Геодезические отображения римановых и аффинно-связных пространств. В сб.: Итоги науки и техники. Сер.: Современная математика и ее приложения. Геометрия-2. Ред. Н. М. Остиану. М.: ВИНИТИ, 1994. Т. 11. С. $112-148$.

[5] Й. Микеш. ДАН СССР. 1989. Т. 305. № 3. С. 534-536.

[6] E. Beltrami. Ann. Mat. 1865. V. 1. № 7.

[7] E. Beltrami. Ann. Mat. 1868. V. 2. № 2. P. 232-255.

[8] P.J. Topalov, V.S. Matveev. Geodesic equivalence and integrability. Preprint series of Max-Planck-Institut Math. № 74. Bonn: MPI, 1998.

[9] E. T. Whittaker. A Treatise on the Analytical Dynamics of Particles and Rigid Bodies. Cambridge: Cambridge University Press, 1937.

[10] И. А. Тайманов. Изв. АН СССР. Сер. матем. 1987. Т. 51. № 2. С. 429-435.

[11] П. Ж. Топалов. Мат. сб. 1997. Т. 188. № 2. С. 137-157.

[12] Н. С. Синююов. ДАН СССР. 1966. Т. 169. № 4. С. 770-772.

[13] S. Tabachnikov. Projectively equivalent metrics, exact transverse line field and the geodesic flow on the ellipsoid. Preprint UofA-R-161. Fayetteville: University of Arkansas, 1998.

[14] А. В. Браилов. Изв. АН СССР. Сер. метем. 1986. Т. 50. № 4. С. 661-674.

[15] P. J. Topalov. Hierarchy of integrable geodesic flows. Preprint series of Max-Planck-Institut Math. № 115. Bonn: MPI, 1998.

[16] A. V. Bolsinov, A. T. Fomenko, V.S. Matveev. Riemannian metrics with integrable geodesic flows on surfaces: local and global geometry. Preprint series of Max-Planck-Institut Math. № 122 . Bonn: MPI, 1998. 